\title{
Hydrologie radar : bilan et perspectives
}

\author{
H. Andrieu \\ J.D. Creutin, G. Delrieu \\ J. Fournet, Y. Pointin
}

\author{
LCPC Nantes \\ IM Grenoble \\ LAMP, OPGC Clermont Ferrand
}

\section{Introduction}

Bien que la mesure des précipitations ait été très tôt reconnue comme l'un des domaines d'application privilégiés de la télédétection radar (la relation de MarshallPalmer liant la réflectivité radar $Z$ à l'intensité de la pluie $R$ a été établie en 1948), il est assez paradoxal de constater que les exemples d'utilisation opérationnelle du radar en hydrologie quantitative demeurent à l'heure actuelle extrêmement rares.

En fait, l'hydrologie radar a connu un essor important depuis le début des années 1970, époque à laquelle les progrès de l'électronique et de l'informatique ont permis d'envisager une véritable exploitation quantitative de la mesure. Cette période a été marquée par un optimisme parfois exagéré vis-à-vis des capacités du radar, présenté comme un instrument devant supplanter à terme les réseaux dans des conditions très favorables avec, parfois, des protocoles de validation insuffisamment rigoureux, expliquent en grande partie cet élan. Depuis le début des années 1980, la littérature radar est plus nuancée, l'intérêt et les limites de la mesure ayant été mieux cernés à la lumière d'expérimentations menées dans des contextes plus variés. L'expérience anglaise, concrétisée par les rapports du Dee Weather Project, 1977 et du North West Weather Radar Project, 1985, nous paraît très révélatrice de cette évolution récente des mentalités.

\section{Mesures hydrologiques par radar}

Le principe de la mesure radar a été présenté en détail dans de nombreux ouvrages (voir par exemple BATTAN, 1973; SAUVAGEOT, 1982), aussi nous limiterons-nous dans ce paragraphe à une revue des principaux facteurs conditionnant selon nous une bonne utilisation de cet instrument de mesure en hydrologie quantitative.

\subsection{Caractéristiques techniques du radar. Eta- lonnage électronique}

La première exigence des hydrologues est de disposer d'une connaissance des précipitations à des échelles de temps et d'espace adaptées à la dynamique des bassins versants d'intérêt. Ces échelles utiles varient évidemment selon les applications (tableau ci-dessous) et ce fait doit être pris en considération dans le choix des caractéristiques techniques du radar.

\begin{tabular}{|c|c|c|}
\hline Application & $\begin{array}{l}\text { Echelles } \\
\text { spatiales }\end{array}$ & $\begin{array}{c}\text { Echelles } \\
\text { temporelles }\end{array}$ \\
\hline $\begin{array}{l}\text { Hydrologie en zone } \\
\text { urbaine }\end{array}$ & qq ha à qq $\mathrm{km}^{2}$ & $5 \mathrm{mn} \quad 1 \mathrm{~h}$ \\
\hline $\begin{array}{l}\text { Hydrologie en région } \\
\text { montagneuse }\end{array}$ & $\begin{array}{l}\text { qq } \mathrm{km}^{2} \text { à qq centaines } \\
\text { de } \mathrm{km}^{2}\end{array}$ & $1 \mathrm{~h}$ \\
\hline $\begin{array}{l}\text { Hydrologie des grands } \\
\text { cours d'eau }\end{array}$ & $\begin{array}{l}\text { qq centaines de } \mathrm{km}^{2} \\
\text { à plusieurs milliers } \\
\text { de } \mathrm{km}^{2}\end{array}$ & $12 \mathrm{~h} \quad 48 \mathrm{~h}$ \\
\hline
\end{tabular}

\section{Hydrological radar : Results and experiments currently being conducted in France}

The works devoted to the hydrological use of radar during the last few years in France have brought to light both the potential concern and the limitation of this measurement technique as regards the quantitative evaluation and the forecast of rainfall. These initial results are based on many experiments specifically devoted to hydrology. Following synthesis about recent researches in France and abroad, the results obtained from the Radar-Cévennes are set forth. Finally, the aim of this experiment is to assess the contribution of the double polarisation measurement technique and the need to integrate a radar in a flood announcement system. 
La puissance d'émission (valeurs typiques : 250 à 1000 $\mathrm{kW}$ ), la durée de l'impulsion (de $1 \mathrm{u}_{\mathrm{s}}$ à $2 \mathrm{u}_{\mathrm{s}}$ ) et la fréquence de répétition des tirs $(250 \mathrm{à} 1000 \mathrm{~Hz})$ déterminent ainsi la portée théorique (200 à $400 \mathrm{~km}$ ) et la résolution radiale de la mesure $(150$ à $300 \mathrm{M})$. L'ouverture du faisceau (1 à $2^{\circ}$ ) et le gain de l'antenne fixent la résolution angulaire et rendent compte de la capacité du radar à concentrer l'énergie émise et rétrodiffusée. Ces deux dernières grandeurs dépendent à la fois de la dimension de l'antenne et de la longueur d'onde d'émission. Une antenne large et une longueur d'onde courte $(3,2 \mathrm{~cm}$ par exemple) permettent d'obtenir un faisceau étroit et un gain important dont il pourrait résulter une mesure fine des faibles intensités à de grandes distances. Cependant, pour une telle longueur d'onde, l'atténuation du faisceau est élevée et l'on admet qu'elle ne devient négligeable que pour une longueur d'onde de $10 \mathrm{~cm}$, avec la contrepartie d'une sensibilité moindre aux faibles intensités.

On voit donc que le choix d'un système radar résulte d'une série de compromis techniques en fonction de l'application projetée auxquelles s'ajoutent diverses considérations économiques : ainsi le coût d'un radar est-il sensiblement proportionnel au poids de l'antenne, soit approximativement à la puissance 3 de la longueur d'onde (Joss et WALDVOGEL, 1987).

D'autre part, une très grande attention doit être portée, pour les applications quantitatives, à l'étalonnage électronique du radar c'est-à-dire à la mesure et au maintien dans le temps des divers paramètres de la mesure. A défaut d'une précision absolue parfaite, la condition minimale requise est la stabilité globale du système de mesure. Ce problème a été discuté dans NWWRP, 1985, Joss et WALDVogel, 1987 et DAVID et al., 1986 , à propos de radars opérationnels depuis quelques années en Angleterre, en Suisse et en France. Moyennant le choix de composants électroniques de qualité et l'utilisation d'un matériel de test précis (et coûteux), les erreurs instrumentales liées au matériel radar peuvent rester dans des limites très raisonnables.

\subsection{La relation $Z-R$}

La relation liant la réflectivité $Z$ - la seule grandeur accessible à la mesure par radar conventionnel - à l'intensité des précipitations $R$ - la grandeur utile pour les applications - a longtemps été considérée comme la source d'erreur principale de la mesure radar. Ce problème a ainsi été abondamment discuté dans la littérature (voir par exemple BATTAN, 1973).

Rappelons que la réflectivité, proportionnelle à la puissance rétrodiffusée, est le moment d'ordre 6 de la distribution granulométrique des gouttes présentes dans le volume échantillonné. L'intensité des précipitations s'exprime aussi en fonction de la distribution granulométrique mais le diamètre des gouttes y intervient à la puissance $3 ; R$ dépend également des conditions de vitesse de vent et de chute des gouttes au sein de la cible. Il n'existe donc pas de relation univoque liant $Z$ à $R$ et l'on doit avoir recours à l'utilisation de relations semi-empiriques dont la forme générale est:

$$
Z=A R^{b} \quad Z\left(\mathrm{~mm}^{6} / \mathrm{m}^{3}\right) \text { et } R(\mathrm{~mm} / \mathrm{h})
$$

où les paramètres $A$ et $b$ varient très sensiblement en fonction de la nature des précipitations.

Atlas et al., 1984, ont chiffré l'importance de ce problème en calculant, à partir de mesures de distribution granulométrique de gouttes au sol, l'écart absolu moyen normé entre les intensités déduites directement de la distribution granulométrique et celles estimées via une relation $Z-R$ donnée. Cet écart, relativement important $(33 \%)$, justifie l'intérêt accordé aux techniques de mesure à paramètres multiples (RoGers, 1984; DoviaK, 1984), l'idée étant d'ajouter à la mesure des réflectivités, la mesure d'une ou deux grandeurs supplémentaires permettant une meilleure estimation de l'intensité des précipitations au sein du volume échantillonné. Parmi ces techniques, la mesure à double polarisation semble être la plus prometteuse mais nécessite encore une évaluation approfondie. Joss et WALDVOGEL, 1987, craignent toutefois que le gain apporté par cette méthode ne reste mineur et que sa mise en œuvre n'induise des effets négatifs sur la stabilité du système de mesure (sophistication et « fragilité " des composants additionnels).

\subsection{Représentativité de la mesure radar par rapport à la pluie au sol}

La transformation des intensités mesurées au sein du volume échantillonné en intensités de pluie au sol nous paraît susceptible d'introduire des erreurs bien supérieures à celles évoquées au paragraphe précédent.

Ce problème est d'autant plus crucial que l'on s'éloigne du radar, la forme du faisceau, le site de tir (angle du faisceau par rapport au plan horizontal) et la courbure de la terre entraînant en effet une augmentation progressive de la taille et de l'altitude du volume échantillonné. De simples considérations géométriques montrent, par exemple, que pour un faisceau de $1^{\circ}$ d'ouverture et un site de tir de $1^{\circ}$, la plus grande partie $\mathrm{du}$ faisceau se trouve au-dessus de $3000 \mathrm{~m}$ (sommet des nuages de type stratiforme) à une distance de $150 \mathrm{~km}$. Ce problème de remplissage partiel du faisceau se pose aussi, mais dans une moindre mesure (l'extension verticale des nuages étant plus importante) en marge des cellules convectives. De plus, la précipitation subit au cours de sa chute d'importantes modifications sous l'action de facteurs microphysiques (coalescence, évaporation...) ou dynamiques (cisaillement du vent, effets orographiques). Certains auteurs (ZAWADZKI, 1984; KoIS. TINEN, 1986) proposent d'estimer, à partir d'explorations volumiques dans les zones proches du radar, un profil vertical moyen de réflectivité qui servira à corriger les mesures réalisées aux plus grandes distances en fonction de l'altitude du volume échantillonné. Il n'existe cependant pas, à notre connaissance, d'étude de validation mettant en évidence le gain apporté par ce mode de correction, et les radaristes anglais (NWWRP, 1985) nous paraissent avoir adopté une attitude prudente et réaliste en limitant la portée hydrologique utile du radar à $75 \mathrm{~km}$.

\subsection{Les phénomènes parasites}

Deux raisons expliquent l'importance fondamentale de ces phénomènes (échos de sol, masques, propagation 
anormale, bande brillante) pour les applications quantitatives: (i) ils rendent le plus souvent inexploitable l'information recueillie sur les zones affectées; (ii) leurs manifestations sont généralement plus intenses là où l'intérêt hydrologique potentiel du radar est le plus grand (zones urbaines, régions montagneuses).

Nous reviendrons au paragraphe 4 sur les méthodes permettant de minimiser l'importance des échos de sol et des masques : choix de l'emplacement du radar, mode de balayage multi-site et définition d'un masque localisant les échos fixes résiduels. Signalons également la méthode proposée par ForTIN et al., 1986 qui peut être utile aussi à la correction des problèmes de propagation anormale. Cette méthode est fondée sur des statistiques de gradients d'intensité en fonction de la distance entre mailles de mesure. Le critère de réjection des mailles affectées est d'autant plus sévère que la zone considérée est sujette "climatologiquement " à des problèmes de phéomènes parasites. Bien que la définition des seuils de réjection paraisse toujours quelque peu subjective, ce type d'approche mérite d'être exploré.

Par ailleurs, si l'identification du phénomène de bande brillante est généralement aisée à partir de coupes verticales de réflectivité (RHI, exploration multi-site), la correction de ce problème demeure pour l'instant extrêmement délicate (SмIтH, 1986). A noter que pour des radars installés en altitude (Joss et VALDVOGEL, 1987), le parasitage induit est très important, le faisceau se trouvant fréquemment et sur des zones larges au niveau de l'isotherme $0^{\circ} \mathrm{C}$.

\subsection{Validation et étalonnage de la mesure radar à l'aide de mesures pluviographiques au sol}

Compte-tenu des différents problèmes que nous venons d'évoquer, il paraît difficile de parler de précision de la mesure radar sans faire explicitement référence à un contexte expérimental donné. Si les erreurs dues à un mauvais étalonnage électronique du radar ou au problème de la relation $Z-R$ sont loin d'être négligeables, celles induites par les modifications des précipitations au cours de leur chute ou par les phénomènes parasite peuvent être d'une ampleur très supérieure.

De plus, le protocole de validation lui-même n'est pas sans influence sur les résultats. Un tel protocole repose sur la définition d'une pluie de référence et l'utilisation de critères d'évaluation adaptés. S'il est naturel d'utiliser la mesure pluviographique (mesure directe au sol) pour le calcul de la référence, encore faut-il se doter de moyens de contrôle de la qualité de cette mesure dont les défauts (erreurs instrumentales, représentativité spatiale) augmentent avec la diminution du pas d'intégration temporel considéré. Un critère d'évaluation important réside dans la comparaison des performances du radar à celles fournies par interpolation des mesures de réseaux sol de différentes densités. Là encore, diverses précautions sont nécessaires afin de garantir l'indépendance entre l'information pluviographique utilisée dans le calcul de la référence et celle utilisée pour l'interpolation. Le lecteur trouvera dans WILSON et BrandEs, 1979; Collier, 1986a et b et Delrieu et al., 1987, les résultats de plusieurs études de validation pour des pas de temps de $1 \mathrm{~h}$ à $24 \mathrm{~h}$.

L'utilisation de mesures pluviographiques a été préconisée pour tenter d'améliorer les performances du radar. Les méthodes proposées vont du simple étalonnage uniforme (WILSON et BRANDES, 1979) à des méthodes plus élaborées visant à une correction régionalisée de l'image radar (BRANDES, 1975; NWWRP, 1985; CreuTIN et $a l ., 1987)$. Bien que des résultats encourageants aient été obtenus, la prudence est nécessaire dans la mise en œuvre de telles approches et cela d'autant plus que l'estimation des facteurs correctifs repose sur un nombre réduit de stations pluviographiques et des pas de temps courts. L'intérêt de l'étalonnage doit être évalué par rapport aux performances du radar non corrigé mais aussi par rapport à celles du réseau d'étalonnage seul, ce qui est assez peu souvent le cas dans la littérature. Enfin, il nous semble que l'étalonnage à l'aide de mesures sol ne saurait se substituer à une critique approfondie et une précorrection des images radars (étalonnage électronique, effets de distance, phénomènes parasites).

\section{Expérimentations en cours en France}

Le réseau ARAMIS de la Météorologie nationale est maintenant dans sa phase terminale d'implantation: les données de 11 radars répartis sur le territoire sont rassemblées tous les quarts d'heures et rediffusées après traitement sur un réseau de consoles spécialisées (Météotel) avec d'autres "produits" météorologiques. Bien que ce dispositif n'ait pas pour vocation première l'hydrologie, la Météorologie nationale favorise et participe à l'adaptation de certains radars du réseau pour mieux répondre à des besoins hydrologiques locaux.

C'est le cas du radar de Trappes pour la surveillance des bassins versants urbains de la DDE Seine St. Denis (ANDRIEU, JACQUET, 1987): les images sont transmises toutes les cinq minutes au système d'aide à la gestion automatisée du réseau d'assainissement. Dans ce contexte expérimental, le GERGRENE poursuit ses recherches portant notamment sur les méthodes et l'impact de la prévision de la pluie sur cette gestion.

On peut citer aussi l'exemple du radar de Mérignac pour lequel une étude est en cours en vue de (i) limiter les problèmes d'échos de sol sur la ville de Bordeaux (ii) réaliser le couplage de l'information radar avec celle des réseaux sol existants (PATAC, réseau de la CUB).

A l'IMG, outre le projet Cévennes $86-88$ que nous décrirons plus en détail au paragraphe suivant, nous étudions le développement d'un radar de courte portée et de coût réduit dévolu à la mesure quantitative sur depetites unités hydrologiques (zones urbaines, bassins versants montagneux...). Dans une première phase, l'évaluation hydrologique d'un radar $3.2 \mathrm{~cm}$ (LARS-88 de EEC) va être réalisée sur l'agglomération grenobloise au début de l'année 1988 .

Enfin, un projet d'utilisation du radar de Grèzes, propriété du Service d'annonce des crues de la Dordogne et récemment intégré au réseau ARAMIS, se développe. Le bassin versant de la Dordogne, qui est remarquable- 


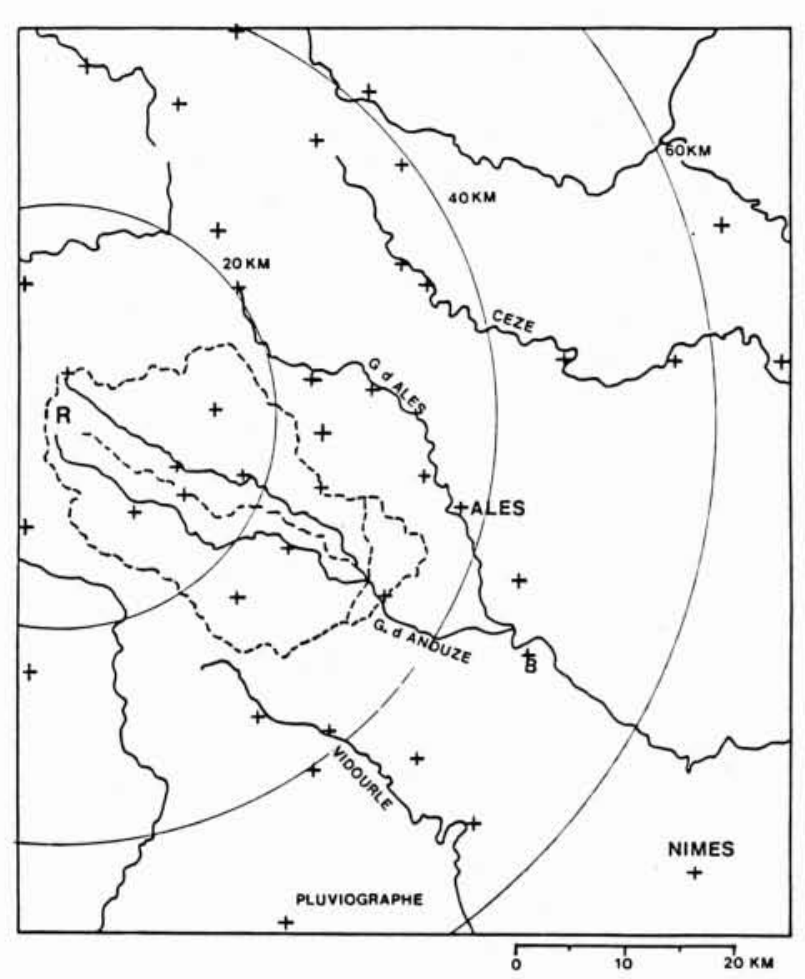

1. Présentation de la zone d'étude.
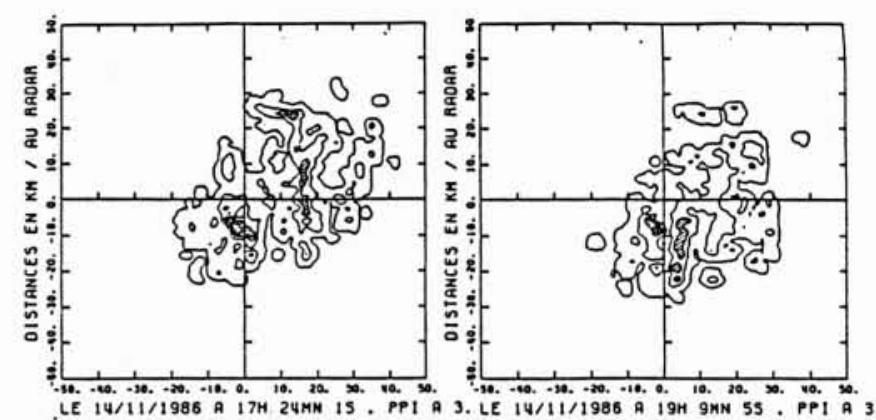

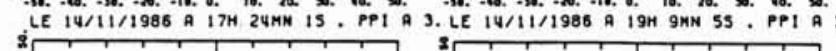
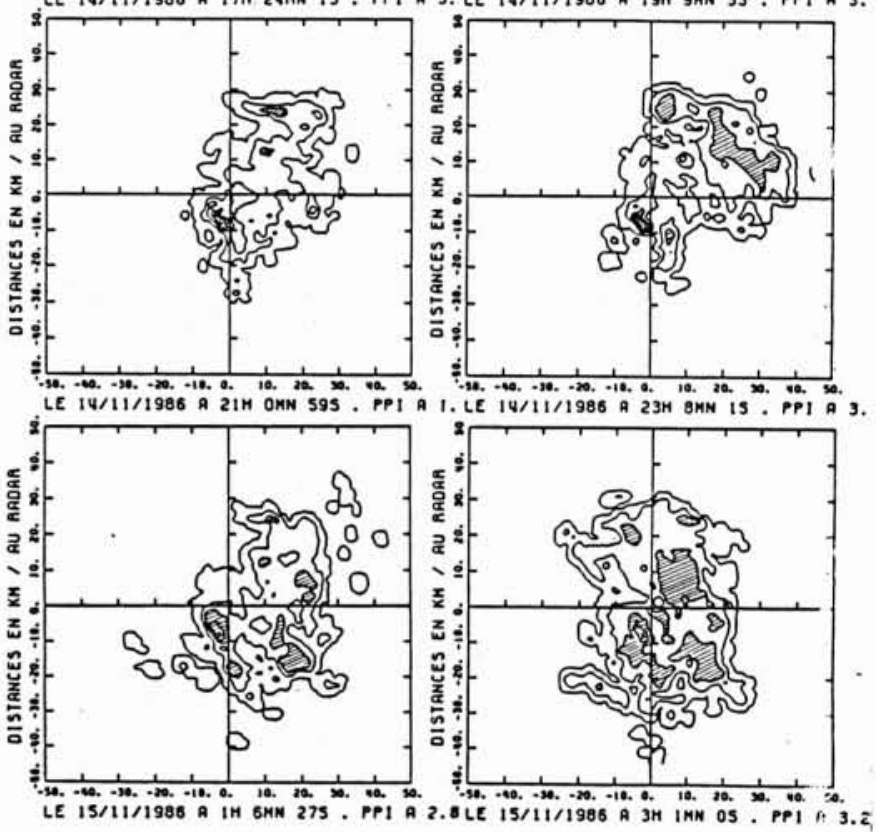

2. Evolution des images radar durant la soirée du 14 au 15 novembre. ment couvert par les deux radars de Grèzes et de Bordeaux, présente tous les atouts pour constituer un bon site d'expérimentation en hydrologie radar.

\section{Expérience Cévennes 86-88}

S'appuyant surle regroupement de plusieurs équipes: IMG, OPGC et LCPC, cette expérience, financée par le Ministère de l'Environnement, se fixe pour objectif de qualifier le radar météorologique pour la mesure des précipitations en hydrologie rurale à des pas de temps de l'ordre de l'heure, en intégrant les possibilités offertes par la diversité de polarisation. L'évaluation de l'intérêt du radar dans un système de crues sera également poursuivie. Cependant, cette seconde étape, passe par l'intégration des images radar au calage des modèles de ruissellement et d'écoulement; elle ne pourra être atteinte qu'a plus longue échéance grâce à l'enregistrement de plusieurs événements pluvieux.
La recherche de conditions expérimentales adaptées aux objectifs poursuivis (paragraphe 2) est l'un des éléments moteurs du projet. Le choix de la région Cévenole répond à ce souci.

Les Cévennes sont régulièrement soumises, durant l'automne, à des pluies intenses et longues à l'origine de crues violentes. Les bassins versants des cours d'eau concernés ont des superficies et des temps de réponse propices à l'utilisation du radar. Devant le risque que représentent les pluies cévenoles, le service d'annonce des crues de la DDE du Gard s'est équipé d'un réseau télétransmis de mesures pluviographiques et limnigraphiques; EDF/DTG dispose également dans cette région de plusieurs points de mesures en cours d'automatisation. Les pluies cévenoles ont, de plus, fait l'objet au cours des dix dernières années d'études suivies, tant sur le plan hydrologiques (IMG, EDF/DTG) que météorologique (LAMP).

L'ensemble de ces éléments, auxquels s'ajoute la disposition d'un radar mobile, fonde un contexte favorable à une expérimentation en hydrologie radar. 


\subsection{Dispositif expérimental et données collec- tées (figure 1)}

Pendant la saison d'automne, le radar ANATOL* de l'OPGC, est installé sur la corniche des Cévennes (à proximité de Barre des Cévennes) à une altitude de $1030 \mathrm{~m}$. Cette implantation est choisie de façon à assurer une bonne couverture des bassins versants des Gardons et du Vidourle. Au cours d'un événement pluvieux les images radar sont acquises au rythme moyen d'une image toutes les deux minutes, en alternant les images à site bas $\left(1^{\circ}\right)$ et à site haute $\left(3^{\circ}\right)$. Outre une mesure classique de réflectivité, le radar donne également une mesure de réflectivité différentielle (rapport des réflectivités dans les deux plans de polarisation); cette dernière quantité apporte une indication sur la taille moyenne des gouttes de pluie. Les données radar, acquises en coordonnées polaires sont transformées en intensités de pluie par la loi $Z=200 R^{1,6}$ et cumulées au pas de temps horaire sur une grille cartésienne dont la maille à 500 $\mathrm{m}$ de côté.

Deux disdromètres, couplés avec des pluviographes, permettent d'enregistrer en permanence la distribution granulométrique des précipitations.

Le réseau de mesure pluviométrique regroupe 45 appareils utilisables au pas de temps horaire et situés dans un rayon d'environ $65 \mathrm{~km}$ autour du radar. Ce nombre sera porté à 50 pour la prochaine saison de mesures. L'utilisation conjointe de données radar et pluviographiques rend nécessaire des mesures de qualité dont la synchronisation en temps soit assurée.

Sont également disponibles les enregistrements limnimétriques télétransmis de la DDE du Gard effectués sur les bassins versants des Gardons, de la Cèze et du Vidourle.

L'automne 1986 a connu deux séquences pluvieuses significatives, dont une seule (du 13 au 15 novembre) présente un caractère cévenol. La première, du 12 au 15 octobre, a été partiellement suivie au radar pendant une durée de $20 \mathrm{~h}$ environ. Au cours de la seconde, du 13 au 15 novembre, 48 heures d'enregistrement radar ininterrompues, qui n'intègrent pas le début de l'événement, ont pu être réalisées. La hauteur d'eau totale, qui a atteint $380 \mathrm{~mm}$ à Villefort, est moins importante dans le sud de la région (73 $\mathrm{mm}$ à St. Hippolyte du Fort). Les intensités horaires ont rarement dépassées 15 à $20 \mathrm{~mm}$ et sont donc restées modérées pour ce type d'événement pluvieux. Les premiers résultats, présentés par la suite seront relatifs à cet épisode.

\subsection{Premiers résultats}

\subsubsection{Analyse des caractéristiques des nuages pré- cipitants}

L'examen des images radar et de leur évolution apporte des éléments sur l'organisation des zones pluvieuses.

* ANATOL : radar de $10 \mathrm{~cm}$ de longeur d'onde et de $250 \mathrm{~kW}$ de puissance, émettant alternativement dans deux plans de polarisation. Ouverture du faisceau principal : $1.8^{\circ}$.
L'altitude maximale des nuages pluvieux avoisine $2500 \mathrm{~m}$; les pluies n'ont donc pas présenté de caractère convectif, ce qui explique les intensités modérées enregistrées. Cette faible altitude maximale altère la portée utile du radar, lui-même étant situé à plus de $1000 \mathrm{~m}$. Sur les cartes de la figure 2 , qui représentent une succession d'images radar, sont distinguées les zones où la réflectivité est supérieure à $30 \mathrm{dBZ}$, et qui sont donc le siège de précipitations significatives. La plus grande part des précipitations semble provoquée par des zones nuageuses de taille relativement faible (quelques kilomètres de diamètre), qui se propagent rapidement du sud vers le nord ( $50 \mathrm{~km} / \mathrm{h}$ environ) et dont la fréquence d'apparition est très variable dans le temps et dans l'espace.

\subsubsection{Représentativité de la mesure radar}

Dans une première étape, la réflectivité différentielle n'est pas utilisée; les données radar sont donc interprétées de façon conventionnelle.

Au cours du paragraphe 2, nous avons insisté sur l'importance que peuvent prendre les phénomènes parasites susceptibles d'affecter l'image. Avant de s'attacher à la mesure des précipitations à l'aide du radar, il est donc nécessaire de critiquer avec soin les images de façon à identifier les anomalies : masques, échos de sol,... et à essayer dans la mesure du possible d'y remédier. Nous nous proposons donc ici d'illustrer les difficultés que l'on peut rencontrer en hydrologie radar en essayant de tester des solutions simples. Cette illustration sera effectuée en s'appuyant sur les images radar relatives à la totalité de la lame d'eau enregistrée au cours de l'événement de novembre 1986.

(i) anomalies affectant les images

Les images radar à site haut et bas sont complétées par la carte des précipitations perçue par le réseau de mesure pluviographique (figure 3 , page suivante). Il apparaît tout d'abord que le radar sous-estime nettement les précipitations (d'un facteur 3,5 environ); ce défaut est dû à un étalonnage électronique défaillant pour cette séquence pluvieuse.

L'image radar à site bas (a) met clairement en évidence la problème des échos de sol. Le massif du Mont-Lozère et le Mont-Aigoual sont les principaux d'entre eux. Les précipitations connaissent une baisse très sensible derrière ces deux obstacles qui interceptent une part non négligeable du faisceau. Il apparaît que, dans toutes les directions (sauf le secteur sud-est), les données radar situées à moins de $20 \mathrm{~km}$ du radar sont inexploitables.

L'image radar à site haut (b) a une allure très différente. La superficie affectée par les échos de sol à proximité du radar est beaucoup moins importante. Remarquons cependant les échos de sol provoqués par les lobes secondaires du faisceau se réfléchissant sur un des versants de la vallée du Gardon de Mialet. La perte de représentativité consécutive à l'augmentation de l'altitude du faisceau est très nette à site haut. Au-delà d'une distance voisine de 42 à $45 \mathrm{~km}$, aucune précipitation n'est détectée. A cette distance, le faisceau sort totalement des nuages pluvieux, ce qui indique un plafond moyen de $1500 \mathrm{~m}$ environ au-dessus du radar. 


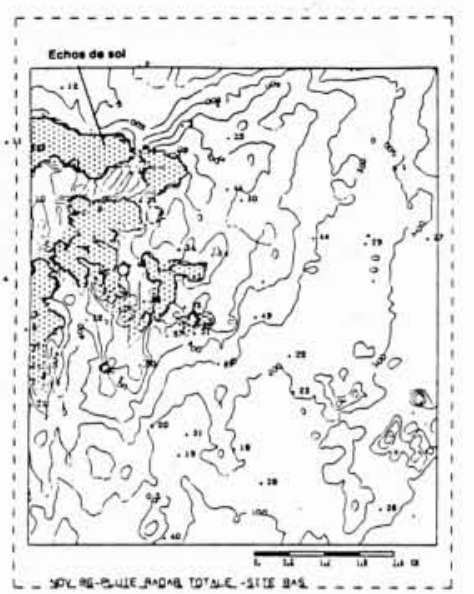

a: Pluile totalo enregistrieg par io
radar a aite bas(1)

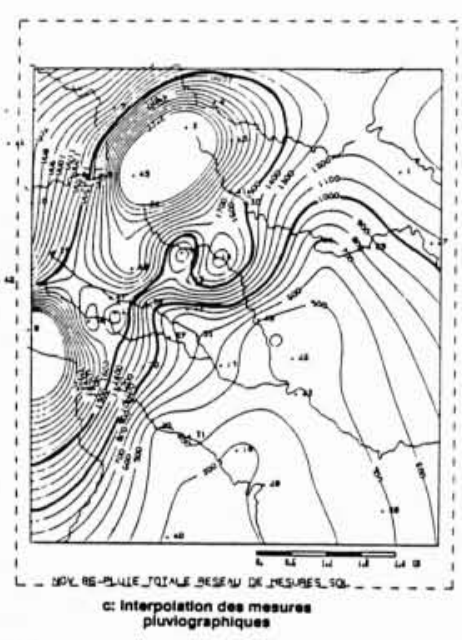

3.

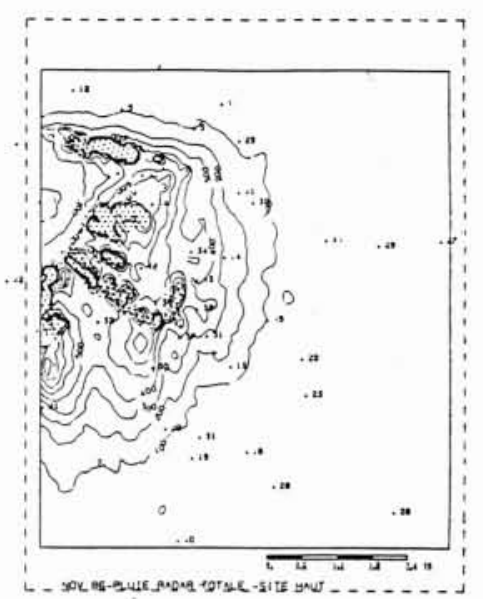

b: Plule totalo onregistee par io
radar a gite haut (3?)

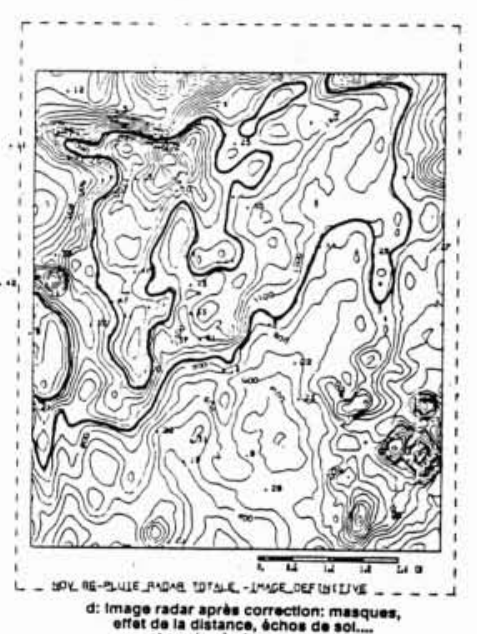

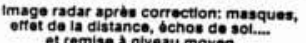
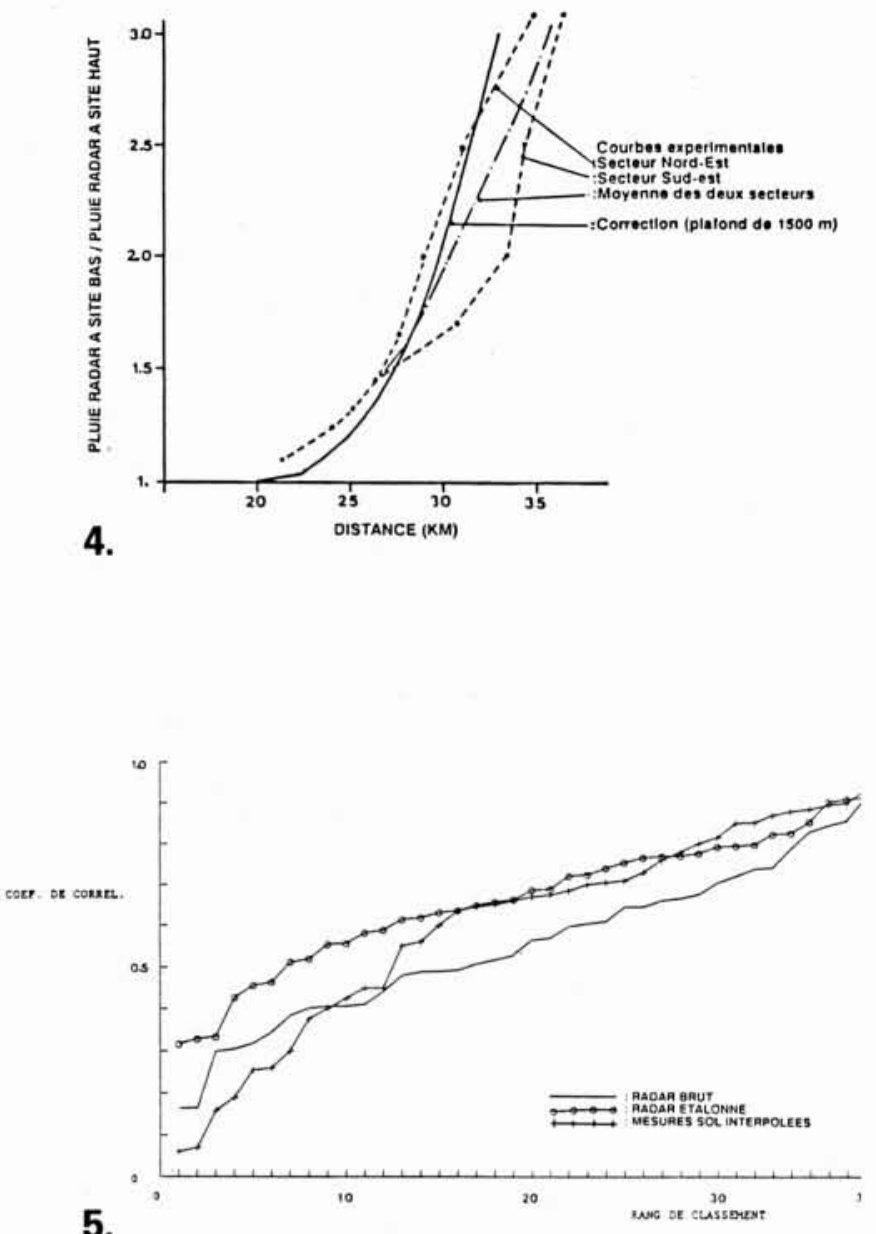

5.

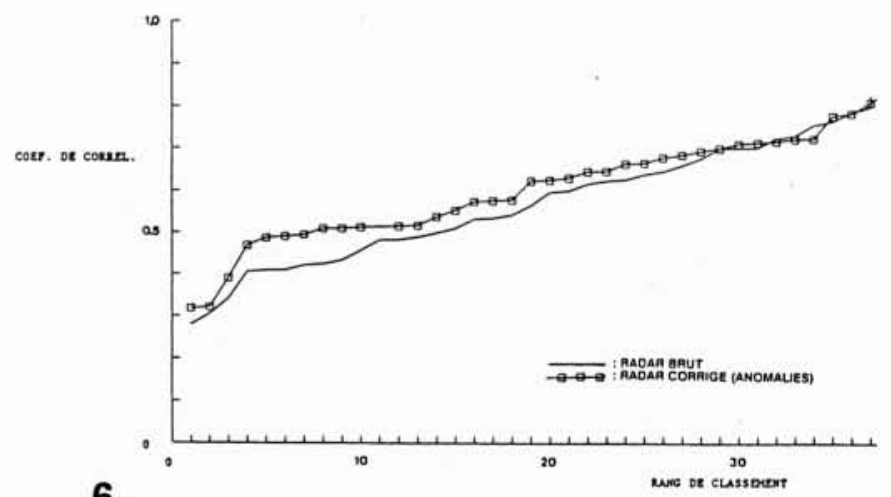

3. Cartographie de la pluie totale tombée en novembre 1988.

4. Evolution du rapport de la pluie radar à site bas et haut avec la distance.

5. Courbes d'efficacité comparant les données radar brutes et corrigées.

6. Courbes d'efficacité comparant les données brutes, étalonnées et l'interpolation des mesures sol.

6. 
La correction des images radar devra donc :

- composer les images à sites haut et bas de façon à minimiser l'importance des échos de sol;

- tenter de compenser la perte de représentativité de la mesure radar avec la distance provoquée par le remplissage partiel du faisceau et les masques.

\section{(ii) Correction des données radar}

Le site haut sera conservé dans la zone la plus proche de façon à réduire les échos de sol, jusqu'à une distance de 20 à $30 \mathrm{~km}$ selon les secteurs. Le problème à résoudre réside dans le remplissage partiel du faisceau, qui débutant à $20 \mathrm{~km}$ pour le site haut, se traduit par une discontinuité à la distance de changement de site.

La figure 4 représente l'évolution moyenne du rapport de la pluie perçue par le radar à site bas et haut, en fonction de la distance, pour deux secteurs de l'image. Cette courbe moyenne peut être utilisée pour corriger l'image à site haut.

Une autre approche, plus générale, est possible. $\mathrm{Si}$ l'on admet que la puissance émise est uniformément répartie dans le faisceau, lorsque seule une proportion $Q$ de la surface du faisceau est concernée par les précipitations, on peut considérer que l'intensité de pluie réelle $R_{R}$ sera :

$$
R_{R}=(1 / Q)^{1 / b} R
$$

$R$ étant l'intensité initialement obtenue par une loi $Z=A R^{b}$. La quantité $Q$ est déduite du plafond des précipitations, du site de tir et de la distance au radar.

Cette fonction de correction des données radar, qui conduit à la courbe représentée sur la figure 4 reproduit correctement les constatations expérimentales. Elle sera retenue pour ajuster les images radar à site haut lors de la constitution de l'image résultante. Elle servira aussi à corriger les effets du remplissage partiel qui prennent effet à une distance supérieure à $45 \mathrm{~km}$ pour le site bas. Enfin, on considèrera également que l'effet des masques peut être compensé par une telle fonction. Dans ce cas, la disposition du relief digitalisé des Cévennes permettra au préalable de préciser la proportion du faisceau intercepté par l'horizon.

Les échos de sol ne peuvent être identifiés avec certitude qu'en disposant d'une série d'images radar sans pluie, ou en cumulant un très grand nombre d'images. Compte tenu des données disponibles, nous nous sommes limités pour l'instant à un calcul du masque par simple seuillage de l'image du cumul de précipitations sur la durée de l'épisode.

(iii) Influence de la correction des images

La pertinence de la correction des images est appréciée en adoptant comme référence mesures observées aux stations pluviographiques disponibles, et en leur comparant les données radar correspondantes brutes et corrigées.

Pour ce qui concerne la pluie totale, nous avons :

- corrélation (sol, radar brut) $=0,65$

- corrélation (sol, radar corrigé) $=0,74$

Au pas de temps horaire, il est possible de calculer un coefficient de corrélation pour chaque champ disponible. La figure 5 présente les courbes d'efficacité correspondantes (courbes de coefficients de corrélation classés).
Au vu de ces deux critères, il apparait que la correction préconisée apporte un gain significatif.

\subsubsection{Premier étalonnage des données radar}

(i) L'étalonnage des mesures radar consiste à utiliser conjointement des images radar et des mesures pluviographiques pour améliorer les valeurs que nous fournit initialement le radar seul.

Une méthode d'étalonnage régionalisé très simple a été adoptée. Elle se base sur la constatation que les rapports : pluie sol/pluie radar, très variables, présentent une organisation spatiale. Elle consiste à établir un champ moyen de facteurs de correction ponctuels, qui sera ensuite appliqué à chacune des heures constitutives de l'épisode pluvieux. Ce champ de correction a été déterminé à partir de la lame d'eau totale enregistrée au cours de l'événement pluvieux, par krigeage des mesures ponctuelles. Cet étalonnage a été appliqué aux images radar composées site haut-site bas non corrigées des effets de remplissage partiel du faisceau par la méthode proposée au paragraphe précédent.

\section{(ii) Evaluation de la méthode}

Elle a été entreprise en comparant les données pluviographiques et les mesures radar étalonnées. Le réseau de pluviographes a été divisé en deux sous-réseaux d'importance équivalente: 19 d'entre eux servant à établir le champ de facteur de correction (réseau d'étalonnage), les autres à évaluer la qualité des résultats obtenus (réseau de validation). Les résultats auxquels conduit l'utilisation du réseau pluviographique seul (interpolation par krigeage) sont calculés à titre de comparaison.

Pour ce qui concerne la pluie totale, il apparaît que l'étalonnage des mesures radar et l'interpolation des mesures sol donnent une qualité d'estimation similaire :

\begin{tabular}{lccc}
\hline & $\begin{array}{c}\text { Mesures } \\
\text { radar } \\
\text { brutes }\end{array}$ & $\begin{array}{c}\text { Mesures } \\
\text { radar } \\
\text { étalonnées }\end{array}$ & $\begin{array}{c}\text { Réseau } \\
\text { pluvio- } \\
\text { graphique }\end{array}$ \\
\hline Corrélation & 0,56 & 0,84 & 0,84 \\
\hline
\end{tabular}

On constate également que le gain procuré par l'étalonnage des données radar est conséquent.

Pas de temps horaire : Les courbes d'efficacité, relatives à chacune des approches testées sont représentées figure 6 . Il s'avère tout d'abord que l'étalonnage simple des mesures radar procure une nette amélioration par rapport aux mesures brutes. La comparaison de l'étalonnage des images radar et de l'interpolation pluviographique met surtout en évidence l'apport du radar pour les champs pluvieux horaires très mal appréhendés à l'aide des pluviographes seuls. 


\section{Conclusion}

Ces premiers résultats encourageants nous semblent pouvoir être améliorés par (i) un raffinement de la méthode de composition et de correction des images radar, (ii) la mise en œuvre de méthodes d'étalonnage à l'aide de données sol plus performantes et (iii) l'inté- gration des mesures de réflectivité différentielle.

La constitution d'une banque de données étoffée est cependant la condition indispensable du succès de cette étude. A ce titre, nous tenons à remercier J. Léoussoff et l'équipe du Service d'annonces des crues de la DDE du Gard, le Service Ressources en eau d'EDF/DTG ainsi que les Centres météorologiques de Nîmes et Marignane pour leur collaboration à cette campagne de mesure.

\section{Références}

ANDRIEU H., JACQUET G., 1987. - Radar météorologique de Trappes et estimation des intensités pluvieuses en Seine-St. Denis. La Houille Blanche, $\mathrm{n}^{\circ} 6$.

Atlas D., C.W. Ulbrich and R. Meneghini, 1974. - The multiparameter remote measurement of rainfall, Radio Science, Vol. 19, n 1 Jan-Feb. 1984, pp. 3-21.

BatTAN L.J., 1973. - Radar Observation of the Atmosphere. The Univ. of Chicago Press, Chicago and London, 324 pp.

BRANDES E.A., 1975. - Optimizing rainfall estimates with the aid of radar. J. Appl. Meteor., 14, pp. 1139-1345.

ColLIER C.G., 1986a. - Accuracy of rainfall estimates by radar. Part. I : Calibration by telemetering raingauges. Journal of Hydrology, 83 (1986) 207-223.

ColLIER C.G., 1986b. - Accuracy of rainfall estimates by radar. Part. II : Comparison with raingauge network. Journal of Hydrology, 83 (1986) 225-235.

Creutin J.D., G. Delrieu and T. Lebel, 1987. - Rain measurement by raingauge-radar combination: A geostatistical approach. Acceté pour publication dans Journal of Atmospheric and Oceanic Technology.

DAVID P., J.P. MUSIEDLAK and P. BISSONIER, 1986. - Utilisation du radar Rodin en pluviométrie, résultats des mesures de 1892. Notes Techniques $\mathrm{n}^{\circ} 12$, Direction de la Météorologie Nationale, Boulogne.

Central Water Planning, 1977. - Dee Weather Radar and Real Time Hydrological Forecasting Project. Report of the Steering Comm. CWPU, Reading.
Delrieu G., A. Bellon, J.D. Creutin, 1987. - Estimation de lames d'eau spatiales à l'aide de données de pluviomètres et de radar météorologique. Accepté pour publication dans Journal of Hydrology.

Fortin J.P., H. Proulx, A. Bellon, 1986. - Utilisation des données d'un radar météorologique pour la simulation des écoulements en rivière à l'aide d'un modèle hydrologique matriciel. Journal of Hydrology, 90, 1987, pp. 327-350.

Joss J., A. WALDVOGEL, 1987. - Precipitation measurement and Hydrology, a Review. 40th Anniversary Conf. on Radar Meteorology, Boston, AMS, 41 p.

KoISTINEN J., 1986. - The effect of some measurement errors on radar-derived Z-R relationships. Prepints 23rd Conference on radar meteorology, Vol. 3, pp. 50-53, AMS, Boston.

Consortium of the North West Weather project, 1985. - North West Weather Radar Project. Report of the Steering Group, $73 \mathrm{p}$.

SAUVAGEOT H., 1982. - Radar Météorologie, Eyrolles Editeur, 296 p.

SмITH C.J., 1986. - The reduction of errors caused by bright bands in quantitative rainfall measurements made using radar. J. Atm. Oc. Tech. 3, pp. 129-141.

WILSON J.W. and E.A. BRANDES, 1979. - Radar measurements of rainfall. A summary. Bull. Am. Met. Soc., 60, 1048-1058.

ZAWADZKI I., 1984. - Factors affecting the precision of radar measurement of rain. Preprints 22th Conference on Radar Meteorology ZURICH, Switzerland, Published by the Am. Met. Soc. Boston, 251-256.

\author{
Adresse des auteurs \\ Monsieur H. Andrieu \\ Messieurs J.D. Creutin et G. Delrieu \\ IMG \\ Domaine universitaire \\ BP 68 \\ 38042 St Martin d'Hères \\ Tél. : (16) 76.44.82.63 \\ Messieurs J. Fournet et Y. Pointin \\ LAMP-OPGC
}


M. MoRel SEYTouX: Quand vous faites la comparaison avec des mesures d'intensité sur les pluviographes, quel pas de temps prenez-vous?

M. ANDRIEU: En ce qui concerne l'expérience Cévennes, des pas de temps horaires.

M. PARSY: D'une part, le graphique R-Z ne comporte pas d'asymptote due à l'effet de miroir, et ce en fonction des caractéristiques de l'appareil. D'autre part, vous semblez ignorer les expériences de Grèze entre 1968 et 1972.

$M$. ANDRIEU: Nous n'ignorons pas l'expérience de Grèze, mais les radars ont beaucoup progressé depuis. La relation $\mathrm{R}-\mathrm{Z}$ a été établie d'après des données spectrogranulométriques.

M. CREUTIN: Nous travaillons avec un radar de longueur d'onde de $10 \mathrm{~cm}$. Les problèmes d'atténuation se rencontrent à partir de $3,2 \mathrm{~cm}$ : C'est le cas étudié à Grenoble avec le radar léger, pour lequel nous espèrons éviter le problème de rideaux de pluie. Le graphe $\mathrm{R}-\mathrm{Z}$ incorpore la fluctuation due à la diversité des distributions granulométriques des gouttes d'eau, mais aussi les erreurs dues à la mesure de $\mathrm{Z}$ et aux transformations qui ont lieu en altitude et au sol. La dispersion est alors plus grande. Nous n'avons pas pu travailler avec des pas de temps inférieurs à la journée, car nous nous trouvions dans des configurations particulières (éloignement du radar, faiblesse des mesures, indigence du réseau « sol ",...). Actuellement, avec l'expérience Cévennes nous pensons pouvoir descendre au pas de temps horaire. Nous n'avons pas encore des données assez soignées pour parler de l'utilité du radar et de la précision de la mesure.

M. PIRCHER: Quelle est en moyenne la différence d'altitude entre les faisceaux radar et les stations au sol sur l'expérience Cévennes?

Comment traitez-vous les effets orographiques éventuels, venant compliquer la liaison entre l'estimation radar des précipitations en altitude et la précipitation au sol ?

M. ANDRIEU: Le radar est à une altitude de $1000 \mathrm{~m}$, les pluviographes entre 300 et $1300 \mathrm{~m}$.

Nous n'avons pas suffisamment de données pour estimer l'effet de certains reliefs, mais nous pensons l'étudier plus tard.
M. ROCHE: Avez-vous des idées précises ou des résultats quantitatifs sur l'utilisation de la double polarisation?

$M$. ANDRIEU: Nous mesurons une réflectivité différentielle qui donne une indication sur la déformation des gouttes d'eau. Comme les gouttes d'eau sont d'autant plus déformées qu'elles sont grosses, cela nous donne une indication sur l'intensité de la pluie qui tombe. La double polarisation semble, en théorie, produire une amélioration.

M. ROCHE: Qu'avez-vous pu faire exactement et quelle sera l'exploitation que l'on pourra faire de la méthode?

M. ANDRIEU: II semble que les conditions d'exploitation sont primordiales. Nous devons tout d'abord arriver à avoir des images horaires mises en forme, très "propres». Ensuite l'OPGC nous fournira une loi intensité-réflectivité qui intègre la réflectivité différentielle. Nous essaierons pour notre part d'évaluer l'amélioration apportée sur le plan hydrologique, au pas de temps horaire, par la réflectivité différentielle, en nous appuyant sur l'analyse d'un grand nombre de données.

M. MOREL-SEYTOUX: Les erreurs relatives de $33 \%$ à $100 \%$ que vous avez présentées, sont-elles sur les valeurs ou sur les logarithmes? (texte reconstitué d'après des notes prises en séance).

\section{DELRIEU: Sur les valeurs elles-mêmes.}

M. MOREL-SEYTOUX: Vous mesurez des intensités instantanées (avec le radar). Par comparaison avec les mesures sur le terrain, pouvez-vous voir si les intensités moyennes diminuent lorsque vous essayez de les calculer sur des périodes de temps de plus en plus grandes?

M. DELRIEU: Les effets d'intégration dans l'espace et dans le temps sont évidemment sensibles. Il se traduisent par un lissage et on peut constater alors une meilleure cohérence entre les mesures au sol et les mesures radar.

$M$. ANDRIEU : La mesure radar est instantanée mais volumique, de l'ordre du $\mathrm{km}^{3}$. Il existe une intégration dans le temps du fait que l'on prend en compte une hauteur assez grande. 
Theorie des Flenves

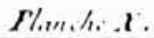

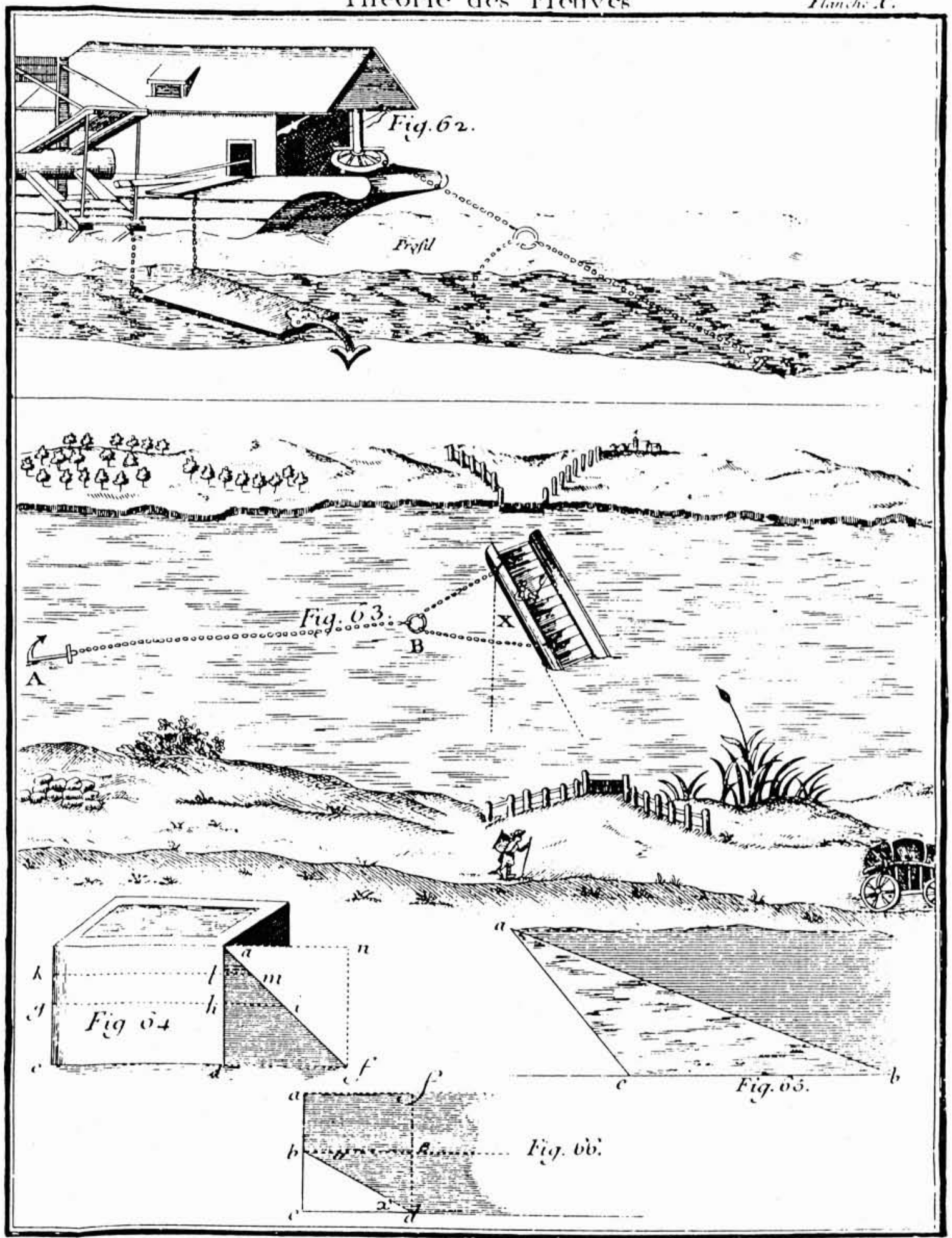

